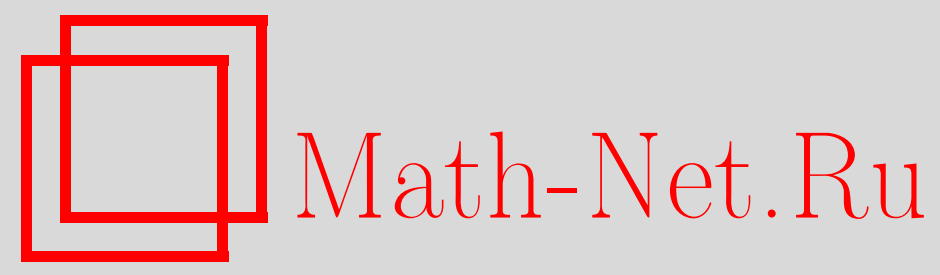

О. П. Виноградов, Вероятность разорения страховой компании в случае, когда интервалы между моментами выплат имеют неодинаковые показательные распределения, Теория вероятн. и ее примен., 1998, том 43, выпуск 2, 352-357

DOI: https://doi.org/10.4213/tvp1470

Использование Общероссийского математического портала Math-Net.Ru подразумевает, что вы прочитали и согласны с пользовательским соглашением http://www.mathnet.ru/rus/agreement

Параметры загрузки:

IP: 54.198 .64 .247

26 апреля 2023 г., $16: 47: 53$

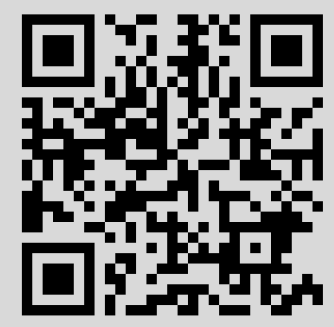




\title{
ВЕРОЯТНОСТЬ РАЗОРЕНИЯ СТРАХОВОЙ КОМПАНИИ В СЛУЧАЕ, КОГДА ИНТЕРВАЛЫ МЕЖДУ МОМЕНТАМИ ВЫПЛАТ ИМЕЮТ НЕОДИНАКОВЫЕ ПОКАЗАТЕЛЬНЫЕ РАСПРЕДЕЛЕНИЯ ${ }^{1}$ )
}

\begin{abstract}
Рассматривается задача нахождения вероятности разорения страховой компании в случае, когда интервалы между выплатами имеют неодинаконые показательные распределения, а величины выплат одинаково распределены и также имеют показательные распределения. В качестве частных случаев рассмотрекы случаи, когда величины выплат неодинаково распределены и имеют эрланговские распределения, а также когда моменты выплат явлются порядковыми статистиками выборки из показательного распределения. Полученные результаты могут найти применение в теории массового обслуживания.
\end{abstract}

Ключевые слова и Фразы: теория риска, теория массового обслуживания, вероятность разорения, двухиндексная последовательность.

1. Введение. В ахтуарной математике хорошо известна задача нахождения вероятности разорения страховой компании. Этой задаче посвящена огромная литература (достаточно полный список литературы можно найти, например, в [1]).

Предположим, что $x>0$ - начальный капитал страховой компании, $S(t)-$ суммарный размер премий, внесенных клиентами за время $[0, t]$. Пусть $T_{1}, T_{2}, \ldots-$ моменты страховых выплат $\left(0 \leqslant T_{1} \leqslant T_{2} \leqslant \cdots\right), Z_{i}-$ размер выплаты в момент $T_{i}$, $\Delta_{i}=T_{i}-T_{i-1}, \Delta_{1}=T_{1}, i \geqslant 1$. Процесс риска $X(t)$ определяется равенством

$$
X(t)=x+S(t)-\sum_{j \leqslant N(t)} Z_{j}
$$

где $N(t)=\max \left(i: T_{i} \leqslant t\right)$ - число выплат компании в интервале времени $[0, t]$. Момент ра́зорения определяется равенством

$$
\tau=\inf (t: X(t)<0) .
$$

Величина $\psi(x)=\mathbf{P}\{\tau<\infty \mid X(0)=x\}$ называется вероятностью разорения.

Мы будем предполагать, что последовательности $\left\{Z_{i}\right\}$ и $\left\{\Delta_{i}\right\}$ независимы и состоят из независимых случайных величин. Процесс $S(t)$ линейно растет на каждом из интервалов $\Delta_{i}$ с угловым коэффициентом $c_{i} \geqslant 0$. Пусть величины $Z_{i}$ одинаково распределены с функцией распределения $1-e^{-x}$, а величины $\Delta_{i}$ также имеют показательное распределение с параметром $\delta_{i}$. Пусть $R(x)=1-\psi(x)$ - вероятность неразорения страховой компании.

В работе найдена формула для вероятности разорения $\psi(x)$. Полученный результат позволяет найти $\psi(x)$ в случае, когда последовательные моменты выплат являются порядковыми статистиками в выборке из показательного распределения; он дает возможность рассмотреть случай, когда величины $Z_{i}$ неодинаково распределены и имеют эрлажовские распределения, а также получить некоторые результаты относительно времени ожидания в одной системе массового обслуживания.

*Механико-математический факультет, МГУ, Воробьевы горы, 119899 Москва, Россия.

1) Работа выполнена при поддержке «The Society of Actuaries Committee on Knowledge Extension Research (CKER)». 
2. Основные результаты. Для нахождения вероятности $R(x)$ и, значит, вероятности $\psi(x)$ предположим сначала, что количество страховых выплат конечно и равно $n$. Тем самым, новый процесс риска отличается от первоначального только числом выплат, а остальные предположения о величинах $\Delta_{i}, Z_{i}, c_{i}, 1 \leqslant i \leqslant n$, остағотя прежними.

Пусть $\psi_{n}(x)$ и $R_{n}(x)$ - соответственно вероятности разорения и неразорения в этом процессе риска с конечным числом выплат. Очевидно, что

$$
\psi(x)=\lim _{n \rightarrow \infty} \psi_{n}(x), \quad R(x)=\lim _{n \rightarrow \infty} R_{n}(\dot{x})
$$

Будем иметь в виду, что

$$
\psi_{n}(x)=\dot{\psi}_{n}\left(x, \delta_{1}, c_{1}, \delta_{2}, c_{2}, \ldots, \delta_{n}, c_{n}\right)
$$

Сделаем в последовательностях $\left\{\delta_{i}\right\}$ и $\left\{c_{i}\right\}, i=1, \ldots, n$, перестановку $\delta_{i} \rightarrow \delta_{n-i+1}$ и $c_{i} \rightarrow c_{n-i+1}$. После такой перестановки интервал времени до первой выплаты будет иметь показательное распределение с параметром $\delta_{n}$, а угловой коэффициент роста капитала на этом интервале будет равен $c_{n}$. Интервал времени между второй и первой выплатой, соответственно, будет иметь показательное распределение с параметром $\delta_{n-1}$, а соответствующий угловой коэффициент будет равен $c_{n-1}$ и т.д. Обозначим через $\widetilde{\psi}_{n}(x)$ и $\widetilde{R}_{n}(x)$ вероятности разорения и неразорения для «переставленного» процесса риска. Очевидно, что из (2) следует, что

$$
\widetilde{\psi}_{n}(x)=\psi_{n}\left(x, \delta_{n}, c_{n}, \delta_{n-1}, c_{n-1}, \ldots, \delta_{1}, c_{1}\right) \text {. }
$$

Таким образом, если найдено явное выражекие для $\widetilde{R}_{n}(x)$ и $\widetilde{\psi}_{n}(x)$, то заменив индексы входяцих в это выражение величин согласно перестановке $i \rightarrow n-i+1$, мы получим выражение для $R_{n}(x)$ и $\psi_{n}(x)$ и, значит, для $R(x)$ и $\psi(x)$ (см. (1)).

Нетрудно видеть, что для всех $i=1, \ldots, n$ справедливы следующцие рекуррентные соотношения:

$$
\widetilde{R}_{i}(x)=\delta_{i} \int_{0}^{\infty} e^{-\delta_{i} y}\left[\int_{0}^{x+c_{i} y} \widetilde{R}_{i-1}\left(x+c_{i} y-z\right) e^{-z} d z\right] d y
$$

причем $\widetilde{R}_{0}(x)=1$.

Полагая $x+c_{i} y=u$, приведем эти равенства к виду

$$
\widetilde{R}_{i}(x)=\beta_{i} e^{\beta_{i} x} \int_{x}^{\infty} e^{-\beta_{i} u}\left[\int_{0}^{u} \widetilde{R}_{i-1}(u-z) e^{-z} d z\right] d u
$$

где $\beta_{i}=\delta_{i} / c_{i}$.

Найти функции $\widetilde{\psi}_{i}(x)=1-\widetilde{R}_{i}(x)$, вероятности разорения, из этих соотношений можно различными способами. На наш взгляд, предложенный ниже подход является одним из наиболее простых.

Положим $\widetilde{\psi}_{i}(x)=\lambda_{i} e^{-x} \alpha_{i}(x)$, где $\lambda_{i}=\beta_{i} /\left(1+\beta_{i}\right)$. Из (3) следует, что функции $\alpha_{i}(x)$ удовлетворяют соотношениям

$$
\alpha_{i}(x)=1+\lambda_{i-1} \int_{0}^{x} \alpha_{i-1}(z) d z+\lambda_{i-1} e^{x / a_{i}} \int_{x}^{\infty} e^{-z / a_{i}} \alpha_{i-1}(z) d z
$$

где $\beta_{0}=\lambda_{0}=0, a_{i}=\left(1+\beta_{i}\right)^{-1}, \alpha_{0}(x)=0, i=1, \ldots, n$.

Легко видеть, что для любого положительного $b$ и всех целых неотрицательных $k$ имеет место равенство

$$
e^{x / b} \int_{x}^{\infty} e^{-x / b} x^{k} d x=k ! \sum_{j=0}^{k} \frac{x_{j} b^{k-j+1}}{j !}
$$


Поэтому из (4) следует, что $\alpha_{i}(x)$ является многочленом степени $i-1$.

Будем искать $\alpha_{i}(x)$ в виде

$$
\alpha_{i}(x)=\sum_{k=0}^{i-1} \widetilde{A}_{i}(k) x^{k}
$$

Таким образом, $\widetilde{A}_{i}(k)=0$ при $k \geqslant i$. Подставляя (6) в (4) и учитывая (5), получаем, что для $i=1, \ldots, n$ справедливы равенства:

$$
\begin{aligned}
& \widetilde{A}_{i}(0)=1+\lambda_{i-1} \sum_{l=0}^{i-2} l ! \alpha_{i}^{l+1} \widetilde{A}_{i-1}(l) \\
& \widetilde{A}_{i}(k)=\frac{\lambda_{i-1}}{\alpha_{i}^{k} k !} \sum_{l=k-1}^{i-2} l ! \alpha_{i}^{l+1} \widetilde{A}_{i-1}(l)
\end{aligned}
$$

для $k=1, \ldots, i-2$ и

$$
\tilde{A}_{i}(i-1)=\lambda_{i-1} \frac{\tilde{A}_{i-1}(i-2)}{i-1} .
$$

Для того, чтобы найти явное выражение для членов двухиндексной последовательности $\left\{\widetilde{A}_{i}(k)\right\}, i=1, \ldots, n, k=0, \ldots, i-1$, приведем соотношения (7)-(9) к более простому виду. Соотношение (8) для $k=1$ принимает вид

$$
\widetilde{A}_{i}(1)=\frac{\lambda_{i-1}}{\alpha_{i}} \sum_{l=0}^{i-2} l ! \alpha_{i}^{l+1} \widetilde{A}_{i-1}(l)
$$

Поэтому из (7) следует равенство

$$
\widetilde{A}_{i}(0)=1+a_{i} \tilde{A}_{i}(1) .
$$

Аналогично, если $k<i-2$, то из (8) следует, что

$$
\tilde{A}_{i}(k+1)=\frac{\lambda_{i-1}}{(k+1) ! \alpha_{i}^{k+1}}\left[\sum_{l=k-1}^{i-2} l ! \alpha_{i}^{l+1} \widetilde{A}_{i-1}(l)-(k-1) ! a_{i}^{k} \widetilde{A}_{i-1}(k-1)\right] .
$$

Отсюда и из (8) получаем, что для $0<k<i-1$

$$
\widetilde{A}_{i}(k)=(k+1) a_{i} \widetilde{A}_{i}(k+1)+\frac{\lambda_{i-1}}{k} \widetilde{A}_{i-1}(k-1) .
$$

Таким образом, мы доказали, что двухиндексная последовательность $\left\{\widetilde{A}_{i}(k)\right\}$ удовлетворяет соотношениям (9)-(11).

Положим для $k>0$

$$
\widetilde{A}_{i}(k)=\frac{\lambda_{i-k} \lambda_{i-k+1} \cdots \lambda_{i-1}}{k !} \widetilde{B}_{i}(k), \quad \widetilde{A}_{i}(0)=\widetilde{B}_{i}(0) .
$$

Тогда соотношения (9)-(11) примут вид

$$
\begin{aligned}
& \widetilde{B}_{i}(0)=1+a_{i} \lambda_{i-1} \widetilde{B}_{i}(1), \\
& \widetilde{B}_{i}(k)=a_{i} \lambda_{i-k-1} \widetilde{B}_{i}(k+1)+\widetilde{B}_{i-1}(k-1)
\end{aligned}
$$

дІІ $0<k<i-1$ и

$$
\widetilde{B}_{i}(i-1)=\widetilde{B}_{i-1}(i-2) .
$$

Так как $\alpha_{0}(x)=0$, то в силу (4) получаем, что $\alpha_{1}(x)=1$. Поэтому

$$
\widetilde{B}_{1}(0)=1 \text {. }
$$


Покажем, что равенства (12)-(15) однозначно определяют двухиндексную последовательность $\left\{\widetilde{B}_{i}(k)\right\}$ и, значит, двухиндексную последовательность $\left\{\widetilde{A}_{i}(k)\right\}$. Действительно, из (14) и (15) следует, что для $i=1, \ldots, n$ справедливо равенство

$$
\widetilde{B}_{i}(i-1)=1 .
$$

Полагая в равенстве (13) $k=i-2$ и используя (16), легко получить, что для $1<i \leqslant n$ справедливо равенство

$$
\widetilde{B}_{i}(i-2)=1+\lambda_{1} \sum_{l=2}^{i} a_{l} .
$$

Используя (13) и (17), аналогичными рассуждениями можно получить явное выражение плх $\widetilde{B}_{i}(i-3)$, если $2<i<n+1$. Действуя аналогично, получаем янное выражение для $\widetilde{B}_{i}(k), i=1, \ldots, n+1, k=0, \ldots, i-1$. Тем самым показано, что равенства (12)(15) однозначно определяют двухиндексную последовательность $\left\{\widetilde{B}_{i}(k)\right\}$.

При помощи элементарных выкладок можно проверить, что последовательность

$$
\begin{aligned}
\widetilde{B}_{i}(k)= & 1+\lambda_{i-k-1} \sum_{l=i-k}^{i} a_{l}+\lambda_{i-k-2} \lambda_{i-k-1} \sum_{l_{1}=i-k}^{i} \sum_{l_{2}=i-k-1}^{l_{1}} a_{l_{1}} a_{l_{2}} \\
& +\cdots+\lambda_{1} \lambda_{2} \cdots \lambda_{i-k-1} \sum_{l_{1}=i-k}^{i} \sum_{l_{2}=i-k-1}^{l_{1}} \ldots \sum_{l_{i-k-1}=2}^{l_{i-k-2}} a_{l_{1}} a_{l_{2}} \cdots a_{l_{i-k-1}}
\end{aligned}
$$

где $\lambda_{p}=0$ при $p \leqslant 0$, удовлетворяет соотношениям (12)-(15) с $1 \leqslant i \leqslant n, 0 \leqslant k \leqslant i-1$. Таким образом, доказана

Теорема 1. Для $n \geqslant 1$ справедливо равенство

$$
\tilde{\psi}_{n}(x)=e^{-x}\left[\sum_{k=0}^{n-1} \frac{\lambda_{n-k} \lambda_{n-k+1} \cdots \lambda_{n}}{k !} \widetilde{B}_{n}(k) x^{k}\right],
$$

где $\widetilde{B}_{n}(k)$ задаются равенствами (18), в которых мужно положить $i=n$.

Сделав перестановку индексов $i \rightarrow n-i+1$ из теоремы 1 мы получим следующую теорему.

Теорема 2. Дая $n \geqslant 1$ справедливо равенство

$$
\psi_{n}(x)=e^{-x}\left[\sum_{k=0}^{n-1} \frac{\lambda_{1} \cdots \lambda_{k+1}}{k !} B_{n}(k) x^{k}\right],
$$

ade

$$
\begin{aligned}
B_{n}(k)= & 1+\lambda_{k+2} \sum_{l=1}^{k+1} a_{l}+\lambda_{k+2} \lambda_{k+3} \sum_{l_{1}=1}^{k+1} \sum_{l_{2}=l_{1}}^{k+2} a_{l_{1}} a_{l_{2}} \\
& +\cdots+\lambda_{k+2} \cdots \lambda_{n} \sum_{l_{1}=1}^{k+1} \sum_{l_{2}=l_{1}}^{k+2} \cdots \sum_{l_{n-k-1}=l_{n-k-2}}^{n-1} a_{l_{1}} a_{l_{2}} \cdots a_{l_{n-k-1}},
\end{aligned}
$$

$\lambda_{p}=0$, eсли $p>n$.

3 а м е ч а н и е 1 . Легко видеть, что

$$
\begin{aligned}
& R_{n}(x)=\mathbf{P}\left\{\max _{1 \leqslant k \leqslant n} \sum_{i=1}^{k}\left(Z_{i}-c_{i} \Delta_{i}\right)<x\right\}, \\
& \widetilde{R}_{n}(x)=\mathbf{P}\left\{\max _{1 \leqslant k \leqslant n} \sum_{i=1}^{k}\left(Z_{n-i+1}-c_{n-i+1} \Delta_{n-i+1}\right)<x\right\} .
\end{aligned}
$$


Из этих равенств, теорем 1 и 2 нетрудно получить формулы для вероятности разорения в случае, когда величины выплат имеют показательное распределение с параметром отличным от 1 .

Следствие 1. Из теорем 1, 2 и замечания 1 вытекает, что двные выражения для $R_{n}(x)$ и $\psi_{n}(x)$ можно получить также в случае, когда величины $Z_{i}$ независимь и имеют разиые распределения, а именяо, их плотность равна $a^{k_{i}+1} x^{k_{i}} e^{-a x} / k_{i}$ ! (распределение Эрланга).

Это утверждение непосредственно следует из соотношений (19), (20) и замечания 1 , если положить некоторые $c_{i}$ равными нулю. Например, если $c_{2}=0$ и $c_{1} c_{3} \neq 0$, то получим выражепие для вероятности разорения в процессе риска, в котором плотность распределения величины первого иска равна $a^{2} x e^{-a x}$.

3 а м е а н и е 2. Полученные результаты дают возможность рассмотреть одну модель теории риска, предложенную в $[2$, с. 41]. Пусть имеется $n$ клиентов страховой компании, принадлежапих одной страховой группе. Промежутки времени до первого несчастного случая для отдельных представителей этой группы можно рассматривать как независимые одинаково распределенные случайные величины с общей функцией распределения $G(x)$. Сообщения о несчастных случаях (или иски) поступают в страховую компанию в порядке возрастания этих промежутков времени. Короче говоря, моменты поступления исков являются порядковыми статистикамн выборки объема $n$ из распределения $G(x)$. Известно [2], что если $G(x)$ непрерывна, то промежутки времени между поступлениями исков независимы тогда и только тогда, когда $G(x)=1-e^{-\delta x}$, причем в этом случае интервалы времени между $i$-м и $(i-1)$-м иском имеют показательное распределение с параметром $(n-i+1) \delta$. В этом случае применима теорема 1 , если положить $\delta_{i}=i \delta$ и сохранить остальные условия этой теоремы.

3 а м е ч а н и е 3. Пусть в однолинейной системе массового обслуживания $W_{n}$ - время ожидания $n$-го требования. Тогда (см., например, [3]) справедливо равенство

$$
W_{n+1}=\max _{1 \leqslant k \leqslant n}\left(0, \sum_{i=1}^{k}\left(T_{i}-Y_{i}\right)\right),
$$

где $T_{i}$ - время обслуживания $i$-го требования, а $Y_{i}$ - интервал времени между моментами поступления $(i+1)$-го и $i$-го требований в систему. Из сравнения $(20)$ и (21) получаем, что если положить

$$
Y_{i}=c_{i} \Delta_{i}, \quad Z_{i}=T_{i},
$$

то $R_{n}(x)=\mathbf{P}\left\{W_{n+1}<x\right\}$. Из этого равенства и предыдуших результатов можно найти распределение времени ожидания произвольного требования при предположениях, вытекаюпцх из равенства (22). А именно, интервалы между поступаюцими требованиями в систему независимы и имеют показательное распределение с разными параметрами, а времена обслуживания разных требований одинаково распределены, независимы и имеют показательное распределение. Обобщение этого результата на случай разнораспределенных времен обслуживания, имеющих эрланговские распределения, можно получить, используя следствие 1.

3 а м е ч а н и е 4. В нашей модели интервалы между выплатами независимы и имеют показательное распределение. На первый взгляд, эти предположения являются очень ограничительными. Однако, если предположить, что $\left\{\delta_{i}\right\}$ и $\left\{c_{i}\right\}$ являются случайными, то мы можем рассмотреть случай зависимых интервалов, которые имеют распределения отличные от показательного.

3 а м е ч н и е 5. Обычно рассматриваются модели, в которых приращение математического ожидания капитала между моментами выплат является положительным. В нашей модели это означает, что $c_{i}>\delta_{i}$ для всех $i$. Если же для некотоpoго $i$ это неравенство не выполнено, то говорят, что $i$-я выплата является большой 
выплатой. Большие выплаты возникают в результате крупных аварий, стихийных бедствий и т.д. и могут привести к разорению страховых компаний. Поэтому модели, которые учитывают большие выплаты, представляют значительный интерес в актуарной математике. Из следствия 1 вытекает, что наша модель может учитывать возможность, когда некоторые из выплат являются большими.

3 а м еч а н и е 6. Известно [4], что функцию распределения произвольной неотрицательной случайной величины с любой точностью можно аппроксимировать в смысле расстояния Леви смесью распределений Эрланга. Этот результат дает возможность в нашей модели приближенно вычислять вероятность разорения, если величины выплат имеют произвольное распределение.

\section{СПИСОК ЛИТЕРАТУРЫ}

1. Калашников В. В., Константияидис Д. Вероятность разорения. - Фундаментальная и прикладная математика, 1996, т. 2, № 4, с. 1055-1100.

2. Галамбош Я. Асимптотическая теория экстремальных порядковых статистик. M.: Наука, 1984.

3. Феллер В. Введение в теорию вероятностей и ее приложения. т. 2. М.: Мир, 1984, $752 \mathrm{c}$.

4. Ковалемко И. H., Филиппова $A . A$. Теория вероятностей и математическая статистика. М.: Высшая школа, 1982, 256 с.

Поступила в редакиию 22.III.1997

(c) $1998 \mathrm{r}$. ДАРХОВСКИЙ Б. С.*

\section{О СТОХАСТИЧЕСКОЙ ЗАДАЧЕ ВОССТАНОВЛЕНИЯ ${ }^{1)}$}

Рассматривается задача восстановления (минимаксного оценивания) линейного функционала при наличии случайных ошибок в наблюдениях. Получена оценка потери качества при использовании линейных методов восстановления. Предлагается также новая формализация содержательной задачи восстановления при случайных ошибках в информации, которая дает возможность во многих случаях эффективно находить оптимальное решение без перехода к асимптотике по шуму.

Ключевые слова и Фразы: минимаксное оцениванне, восстановление линейного функционала.

1. Пусть $X$ - линейное нормированное пространство, $X^{\prime}-$ его сопряженное, $W \subset X$ - выпуклое, центрально-симметричное множество, $x^{\prime} \in X^{\prime},\left\langle x^{\prime}, x\right\rangle$ - значение линейного функционала на элементе $x$. Пусть $Y$ - линейное нормированное

\footnotetext{
*Институт системного анализа РАН, пр. 60-летия Октября, 9, 117312 Москва, Россия.

1)Работа поддержана Российским фондом фундаментальных исследований, грант № 98-01-00962.
} 